\title{
Cognitive Status in COVID-19 Survivors with Metabolic Syndrome
}

\author{
Halder $\mathbf{S}^{1 *}$, Samajdar $\mathbf{S}^{2}$ and Mahato $\mathbf{A K}^{1}$ \\ ${ }^{1}$ Associate Professor, Amity University, Kolkata, India \\ ${ }^{2}$ Clinical Psychologist, Kolkata, India
}

*Correspondence: Susmita Halder, Associate Professor, Amity University, Kolkata, India

Received on 14 June 2021; Accepted on 14 July 2021; Published on 20 July 2021

Copyright (C) 2021 Halder S, et al. This is an open access article and is distributed under the Creative Commons Attribution License, which permits unrestricted use, distribution, and reproduction in any medium, provided the original work is properly cited.

\begin{abstract}
In the current COVID-19 pandemic, co-morbid metabolic syndromes are identified as important risk factors. The presence of metabolic syndrome not only increases mortality rate and probability of hospitalization but is also predicted to have a long-term impact on cognition. A decline in cognitive functioning and functional abilities can be seen in the COVID-19 survivors and it became accelerated by metabolic syndromes that include hypertension, diabetes mellitus, low HDL cholesterol, abdominal obesity, etc. In the present study, it was aimed to explore the cognitive status in COVID-19 survivors with and without metabolic syndrome. For this purpose, total 36 COVID-19 survivors participated who were divided into two groups, one with existing metabolic syndrome and the other without any significant co-morbidities. Mini-Mental State Examination (MMSE) and Brief Cognitive Rating Scale (BCRS) were administered to assess the cognitive status of the participants. Results suggest a significant difference between the groups in the domains of concentration and memory along with functioning and self-care.
\end{abstract}

Keywords: COVID, metabolic syndrome, cognitive functioning, memory

Abbreviations: MMSE: Mini-Mental State Examination; BCRS: Brief Cognitive Rating Scale; MCI: mild cognitive impairment

\section{Introduction}

Coronavirus outbreak has become a public health concern and a global threat. Pandemic situation due to the spread of the COVID-19 virus interrupted the physical, emotional, and social functioning of every individual [1, 2]. Epidemiological studies suggest that the risk of fatality due to the spread of the COVID-19 infection is associated with the rate for the presence of co-morbidity, along with increased age [3]. In co-morbid medical conditions, patients with cardiovascular, chronic respiratory diseases and metabolic syndromes are at the highest risk in terms of getting infected, presenting severity and even being fatal [4-6]. Metabolic syndrome that includes hypertension, diabetes, obesity, and LDL cholesterol is indicative of the increased risk of cardiovascular problems and increase the proneness 
of being severely affected by coronavirus infections. It has been said that obesity and other cardio-metabolic symptoms have an adverse impact in the long-term, and it escalates the inflammatory pulmonary infections resulted in COVID19 conditions [7]. Metabolic syndrome also predicts cognitive impairment as it is evident in obesity, hypertension, and type-2 diabetes [8]. These conditions could enhance the chances of vascular dementia and Alzheimer's disease. Chronic metabolic syndromes also lead to mild cognitive impairment (MCI), and in the long-term turn into dementia and worsen the quality of life. Diabetes, in particular, has been interrelated with poor cognitive function and suggested as a pathogenetic mechanism of impaired cognitive functioning [9-11]. In diabetes, insulin resistance resulting in cognitive impairment, causes severe damage to memory functioning. In the case of cholesterol imbalance, high HDL cholesterol level maintains and protects hippocampal atrophy, improves the synaptic growth and regeneration, and also prevents inflammation, whereas low HDL indicates disturbed memory functions.

In COVID-19 symptoms manifestations, inflammatory cytokines, and C-reactive protein (CRP) have a significant role, and inflammatory activation decreases cognitive functioning, also involved in pathological process of development of MCI [12]. Neuropsychological findings suggest that, COVID-19 patients might have an impairment in the domains of attention, memory, executive functioning, visuo-spatial processing, psychomotor functions, and information processing. Metabolic syndromes and systematic inflammatory response together cause changes in cerebral glucose metabolism and peri-infectious encephalopathy which is associated with the neuropsychological sequalae in COVID patients [13]. These background findings suggest that the presence of metabolic syndromes significantly increases the vulnerability to cognitive impairment when infected by coronavirus. The present study is aimed to explore the cognitive status, including memory domains of COVID-19 survivors with metabolic syndrome.

\section{Materials and Methods}

\section{Participants}

In the present study, total 36 individuals of both sexes were selected with purposive sampling method. They were equally divided into two groups, i.e., COVID-19 survivors with metabolic syndrome (Group A) and without metabolic syndrome (Group B). The age range of the sample was between 18-60 years; and mild to severe level of infection was considered to include in the study. Individuals with existing psychological and neurological disorders were excluded from the sample. Ethical consideration was maintained, and informed consent was taken from each participant prior to the data collection.

\section{Tools used}

- Socio-demographic datasheet: Tailor-made socio-demographic and clinical datasheet was prepared prior to the data collection, and it was given to each participant to collect information regarding age, sex, education, COVID-19 infection and severity, and presence of any other medical condition.

- Mini-Mental State Examination: The Mini-Mental State Examination (MMSE) was used to screen the presence of cognitive impairment among participants [14].

- $\quad$ Brief Cognitive Rating Scale: The Brief Cognitive Rating Scale (BCRS) was used to assess functional and cognitive abilities. The BCRS includes ratings of a number of domains objectively that include different cognitive functions. On this scale, higher scores indicate higher cognitive impairment [15].

\section{Statistical analysis}

In the present study, data were analysed by descriptive statistics and t-test. To compare the data between the groups, a small sample independent t-test was used. Equal variances between the two means were considered as t-test assumes the equal variances and checked by Levene's test of equality of variances. For data analysis, IBM SPSS version 17 was used in this study. 


\section{Results}

\begin{tabular}{|c|c|c|c|}
\hline \multicolumn{2}{|l|}{ Variables } & $\begin{array}{c}\text { Group A }(\mathbf{n}=18) \\
(\text { Mean } \pm \text { SD })\end{array}$ & $\begin{array}{l}\text { Group B }(\mathbf{n}=18) \\
(\text { Mean } \pm \text { SD })\end{array}$ \\
\hline \multicolumn{2}{|l|}{ Age } & $52.58 \pm 10.85$ & $30.27 \pm 11.50$ \\
\hline & & \multicolumn{2}{|c|}{ Number (Percentage) } \\
\hline \multicolumn{4}{|l|}{ Gender } \\
\hline & Male & $8(44.44 \%)$ & $7(38.88 \%)$ \\
\hline & Female & $10(55.55 \%)$ & $11(61.11 \%)$ \\
\hline \multicolumn{4}{|c|}{ Severity of COVID infection } \\
\hline & Moderate & $5(27.77 \%)$ & $7(38.88 \%)$ \\
\hline & Moderately severe & $7(38.88 \%)$ & $8(44.44 \%)$ \\
\hline & Severe & $6(33.33 \%)$ & $3(16.66 \%)$ \\
\hline \multicolumn{4}{|c|}{ Metabolic syndromes $(n=18)$} \\
\hline & Hypertension & $6(33.33 \%)$ & \\
\hline & Diabetes mellitus & $3(16.67 \%)$ & \\
\hline & Abdominal obesity & $2(11.11 \%)$ & \\
\hline & Low HDL cholesterol & $2(11.11 \%)$ & \\
\hline & Diabetes + hypertension & $6(33.33 \%)$ & \\
\hline
\end{tabular}

Table 1: Socio-demographic and clinical details of the sample.

\begin{tabular}{|l|l|l|l|l|l|}
\hline Measures & Sub-domains & $\begin{array}{l}\text { Group A (n= 18) } \\
(\text { Mean } \pm \text { SD) }\end{array}$ & $\begin{array}{l}\text { Group B (n= 18) } \\
\text { (Mean } \pm \text { SD) }\end{array}$ & t-value & p-value \\
\hline Mini-Mental State Examination (MMSE) & $24.05 \pm 2.41$ & $25.55 \pm 2.12$ & $-2.03^{*}$ & 0.05 \\
\hline \multirow{4}{*}{$\begin{array}{l}\text { Brief Cognitive Rating } \\
\text { Scale (BCRS) }\end{array}$} & Concentration & $2.25 \pm 0.85$ & $1.22 \pm 0.64$ & $4.15^{* *}$ & $<0.001$ \\
\cline { 2 - 6 } & Recent memory & $2.10 \pm 0.91$ & $1.27 \pm 0.57$ & $3.28^{* *}$ & 0.002 \\
\cline { 2 - 7 } & Past memory & $1.65 \pm 0.75$ & $1.27 \pm 0.57$ & 1.71 & 0.09 \\
\cline { 2 - 7 } & Orientation & $1.15 \pm 0.36$ & $1 \pm 0.0$ & 1.73 & 0.09 \\
\cline { 2 - 7 } & $\begin{array}{l}\text { Functioning and self- } \\
\text { care }\end{array}$ & $2 \pm 1.02$ & $1.16 \pm 0.38$ & $3.24^{* *}$ & 0.002 \\
\hline
\end{tabular}

Table 2: Comparison of cognitive functioning between COVID-19 survivors with metabolic syndrome (Group A) and without metabolic syndrome (Group B). "Higher scores in MMSE indicate better cognitive functioning and higher scores in BCRS indicate lower cognitive functioning. "p-value $<0.05 ;{ }^{* *}$ p-value $<0.01$ using t-test for group comparison. SD: standard deviation.

\section{Discussion}

In the present study, to understand the cognitive status after COVID infection, individuals infected with the COVID19 virus were included. Further comparisons were made between the COVID survivors with and without presence of metabolic syndrome. The table showed that the mean age of the Group A i.e., COVID survivors with metabolic syndrome is higher than those without any presence of metabolic syndrome, which suggests that, the presence of metabolic syndrome in COVID-19 survivors is more prevalent in higher age group (Table 1). It has been seen that the presence of co-morbidity with metabolic disorders is more prevalent in the age range of 42-55 years [16]. Severity wise distribution was almost similar in both the groups, number of severely infected individuals is found to be slightly higher in Group A. It has been found in the Group A that there were five subtypes of metabolic syndrome that were present in the current sample i.e., hypertension (6), diabetes (3), low HDL cholesterol (2), obesity (2), and diabetes + hypertension (6) conditions.

Cognitive status was assessed by MMSE and BCRS, where overall findings suggest that the COVID survivors with metabolic syndrome (Group A) showed poor cognitive functioning when compared with the survivors without any metabolic syndrome (Group B). Findings also suggest that a significant difference in MMSE has been found between the two groups (t-value -2.03; p-value 0.05) and it indicates better cognitive status among individuals without metabolic syndromes (Group B) (Table 2). In BCRS, a significant difference has been found in the sub-domains of 
concentration (t-value 4.15; p-value 0.000), recent memory (t-value 3.28; p-value 0.002), functioning and self-care (tvalue 3.24; p-value 0.002) in both the levels of critical values. Results suggest that the presence of metabolic syndromes in COVID survivors had detrimental effect on global cognitive functioning and difficulties in sustaining attention along with short-term memory functioning.

Previous research findings also showed that memory functioning is most affected in metabolic syndrome. Metabolic effects such as hyperglycemia, hyperinsulinaemia, biogenic dysfunction and inflammation have direct impact upon hippocampal circuit which is associated with memory functioning [8, 17]. Glucose deregulation causes cerebral atrophy and hormonal changes which leads to altered signal transduction and decreasing the concentration capacity among COVID-19 survivors. Vasoconstriction related to hypertension and inflammation leads to impairment in attention, concentration and information processing. COVID-19 virus adversely affects olfactory and gustatory functions which increase neocortical dysfunction which leads to dysfunction in sensory perception, generation and motor co-ordination and spatial reasoning [18]. The present study clearly shows the significant difficulty in recent memory across the metabolic conditions in COVID survivors.

It is already established that the cognitive decline is associated with the inflammatory process in COVID-19 patients. Patients with co-morbid metabolic syndromes might have more inflammation and it could affect the frontal lobe functioning i.e., sustained attention, memory, and executive functioning [19]. Recent researches were also trying to predict the long-term impact of COVID-19 virus in neurocognitive and psychological functioning of the individuals [20]. Changes in nature of the virus and level of infection may make the condition more complicated. The current study indicates the difficulties in cognitive functioning, which needs to be properly identified and managed, before it becomes more complicated [21].

The small sample size could be a limitation of the study. However, considering the study aimed to explore the acute impact of COVID infection on cognitive functions and if it was more evident in individuals with co-morbid metabolic syndrome conditions, it was a bit difficult to work with a large sample within the available time-span. Moreover, waiting for consent for long could have resulted in waning of the impact of COVID infection on cognitive functions which we intended to measure. Many individuals who were in phase of recovery were hesitant and declined to give consent for the study, as it was a crisis moment for them. The study gives a prospect of studying COVID infected individuals, in a larger sample on similar lines to see the long-term impact of COVID infection on cognitive functioning.

\section{Conclusion}

Changes in cognitive functioning are prominent among survivors of COVID-19 infection due to cytokine storm and inflammation. Presence of metabolic disorders increase the risk of severity and mortality of COVID-19 condition along with deteriorated cognitive functioning. Findings of the present study indicated overall poor cognitive functioning in COVID 19 survivors with metabolic syndrome, especially in the domain of memory. Long-term followup is suggested to identify further deterioration and early intervention should be planned focusing on cognition, emotion, and functional abilities.

\section{References}

1. Zhang C, Yang L, Liu S, et al. Survey of insomnia and related social psychological factors among medical staff involved in the 2019 novel coronavirus disease outbreak. Front Psychiatry. 2020;11:306.

2. Halder S, Mahato A, Manot S. COVID-19: Psychological impact and psychotherapeutic intervention. EC Psychology and Psychiatry. 2020;9(6):32-35.

3. Nandy K, Salunke A, Pathak SK, et al. Coronavirus disease (COVID-19): A systematic review and metaanalysis to evaluate the impact of various comorbidities on serious events. Diabetes Metab Syndr.. 2020;14(5):1017-025. 
4. Badawi A, Ryoo SG. Prevalence of comorbidities in the Middle East respiratory syndrome coronavirus (MERS-CoV): a systematic review and meta-analysis. Int J Infect Dis. 2016;49:129-33.

5. Zhou Y, Chi J, Lv W, et al. Obesity and diabetes as high-risk factors for severe coronavirus disease 2019 (Covid-19). Diabetes Metab Res Rev. 2021;37(2):e3377.

6. Parohan M, Yaghoubi S, Seraji A, et al. Risk factors for mortality in patients with Coronavirus disease 2019 (COVID-19) infection: a systematic review and meta-analysis of observational studies. Aging Male. 2020;25(5): 1416-424.

7. Bornstein SR, Dalan R, Hopkins D, et al. Endocrine and metabolic link to coronavirus infection. Nat Rev Endocrinol. 2020;16(6):297-98.

8. Siervo M, Harrison SL, Jagger C, et al. Metabolic syndrome and longitudinal changes in cognitive function: a systematic review and meta-analysis. J Alzheimers Dis. 2014;41(1):151-61.

9. Akbari E, Asemi Z, Kakhaki DR, et al. Effect of probiotic supplementation on cognitive function and metabolic status in Alzheimer's disease: a randomized, double-blind and controlled trial. Front Aging Neurosci. 2016;8:256.

10. Halder S, Samajdar S, Mahato AK. An exploratory study of cognitive functioning and psychological wellbeing in middle-aged adults with diabetes mellitus. Journal of Social Health and Diabetes. 2020;8(01):00812 .

11. Halder S, Samajdar S, Mahato AK. Impact of age on cognitive and psychological functioning in patients with diabetes. Series Endo Diab Met. 2021;3(1):27-32.

12. Collier ME, Zhang S, Scrutton NS, et al. Inflammation control and improvement of cognitive function in COVID-19 infections: is there a role for kynurenine 3-monooxygenase inhibition? Drug Discov Today. 2021;26(6):1473-481.

13. Komulainen P, Lakka TA, Kivipelto M, et al. Metabolic syndrome and cognitive function: a populationbased follow-up study in elderly women. Dement Geriatr Cogn Disord. 2007;23(1):29-34.

14. Folstein MF, Folstein SE, McHugh PR. "Mini-mental state". A practical method for grading the cognitive state of patients for the clinician. J Psychiatr Res. 1975;12(3):189-98.

15. Reisberg B, Ferris SH. Brief cognitive rating scale (BCRS). Psychopharmacol Bull. 1988;24(4):629-36.

16. Jiang B, Zheng $\mathrm{Y}$, Chen $\mathrm{Y}$ et al. Age and gender-specific distribution of metabolic syndrome components in East China: role of hypertriglyceridemia in the SPECT-China study. Lipids Health Dis. 2018;17(1):92.

17. Hosp JA, Dressing A, Blazhenets G, et al. Cognitive impairment and altered cerebral glucose metabolism in the subacute stage of COVID-19. Brain. 2021;144(4):1263-276.

18. Huang C, Mattis P, Tang C, et al. Metabolic brain networks associated with cognitive function in Parkinson's disease. Neuroimage. 2007;34(2):714-23.

19. Vintimilla R, Hall J, Johnson L, et al. The relationship of CRP and cognition in cognitively normal older Mexican Americans: a cross-sectional study of the HABLE cohort. Medicine (Baltimore). 2019;98(19):e15605. 
20. Palmer K, Monaco A, Kivipelto M, et al. The potential long-term impact of the COVID-19 outbreak on patients with non-communicable diseases in Europe: consequences for healthy ageing. Aging Clin Exp Res. 2020;32(7):1189-194.

21. Zarrabian S, Hassani-Abharian P. COVID-19 pandemic and the importance of cognitive rehabilitation. Basic Clin Neurosci. 2020;11(2):129-132. 